\title{
hNTCP-expressing primary pig hepatocytes are a valuable tool for investigating hepatitis $B$ virus infection and antiviral drugs
}

\author{
MING ZHOU ${ }^{1,2^{*}}$, BO QIN $^{3 *}$, XUE-SONG DENG ${ }^{4}$, XIAO-LI ZENG ${ }^{5}$, YING LU $^{1}$, \\ ZI-GANG HUANG ${ }^{2}$, CHUN-CHEN WU ${ }^{6}$ and LI-SHA MOU ${ }^{1}$
}

\begin{abstract}
${ }^{1}$ Shenzhen Xenotransplantation Research and Development Center, Institute of Translational Medicine, Health Science Center, Shenzhen University School of Medicine, Shenzhen Second People's Hospital, The First Affiliated Hospital of Shenzhen University, Shenzhen, Guangdong 518035; ${ }^{2}$ Liver-Biotechnology (Shenzhen) Co., Ltd., Shenzhen, Guangdong 518110; ${ }^{3}$ Clinical Laboratory Center, Shaoxing People's Hospital, Shaoxing Hospital of Zhejiang University, Shaoxing, Zhejiang 312000; ${ }^{4}$ Department of Hepatobiliary Surgery, Shenzhen Second People's Hospital, The First Affiliated Hospital of Shenzhen University, Shenzhen, Guangdong 518035;

${ }^{5}$ Department of Internal Medicine, The Second People's Hospital of Futian District, Shenzhen, Guangdong 518049; ${ }^{6}$ Key Laboratory of Virology, Wuhan Institute of Virology, Chinese Academy of Sciences, Wuhan, Hubei 430071, P.R. China
\end{abstract}

Received January 28, 2019; Accepted July 24, 2019

DOI: $10.3892 / \mathrm{mmr} .2019 .10628$

\begin{abstract}
Primary human hepatocytes (PHHs) are the 'gold standard' for investigating hepatitis B virus (HBV) infection and antiviral drugs. However, poor availability, variation between batches and ethical issues regarding PHHs limit their applications. The discovery of human sodium taurocholate co-transporting polypeptide (hNTCP) as a functional HBV receptor has enabled the development of a surrogate model to supplement the use of PHHs. In the present study, the evolutionary distance of seven species was assessed based on single-copy homologous genes. Based on the evolutionary distance and availability, $\mathrm{PHHs}$ and primary rabbit hepatocytes (PRHs) were isolated and infected with hNTCP-recombinant lentivirus, and susceptibility to HBV infection in the two cell types was tested and compared. In addition, HBV infection efficiency of hNTCP-expressing PPHs with pooled HBV-positive serum and purified particles was determined. The potential use of HBV-infected hNTCP-expressing PPHs for drug screening was assessed. The results demonstrated that pigs and rabbits are closer to humans in the divergence
\end{abstract}

Correspondence to: Professor Li-Sha Mou or Dr Ming Zhou, Shenzhen Xenotransplantation Research and Development Center, Institute of Translational Medicine, Health Science Center, Shenzhen University School of Medicine, Shenzhen Second People's Hospital, The First Affiliated Hospital of Shenzhen University, 3002 Sungang West Road, Futian, Shenzhen, Guangdong 518035, P.R. China

E-mail: lishamou@gmail.com

E-mail: zhouming2004@126.com

*Contributed equally

Key words: hepatitis B virus, sodium taurocholate co-transporting polypeptide, primary pig hepatocyte, cell model tree compared with mice and rats, indicating that pigs and rabbits were more likely to facilitate the HBV post-entry lifecycle. Following hNTCP complementation and HBV infection, PPHs and Huh7D human hepatocellular carcinoma cells, but not PRHs, exhibited increased hepatitis B surface antigen and hepatitis B e-antigen secretion, covalently closed circular DNA formation and infectious particle secretion. hNTCP-expressing PPHs were susceptible to infection with $\mathrm{HBV}$ particles purified from pooled $\mathrm{HBV}$-positive sera, but were poisoned by raw HBV-positive sera. The use of HBV-infected hNTCP-expressing PPHs for viral entry inhibitor screening was revealed to be applicable and reproducible. In conclusion, hNTCP-expressing PPHs may be valuable tool for investigating HBV infection and antiviral drugs.

\section{Introduction}

Hepatitis B virus (HBV) infection exhibits a wide spectrum of clinical liver manifestations (1). Primary human hepatocytes (PHHs) are currently considered to be the 'gold standard' cell model for studying HBV infection (2). However, poor availability, variation between batches and ethical issues regarding PHHs limit their application. Primary hepatocytes from non-human primates, such as chimpanzees and monkeys, are expensive, difficult to obtain and also associated with ethical concerns (3). Primary tupaia hepatocytes (PTHs) from the treeshrew genus Tupaia belangeri are less expensive, but also difficult to obtain, and HBV infection of PTHs is different compared with that of PHHs, as the addition of dimethyl sulfoxide (DMSO) plus polyethylene glycol 8000 (PEG8000) during infection does not significantly promote HBV infectivity (3).

The discovery of the HBV functional receptor human sodium taurocholate co-transporting polypeptide (hNTCP) (4) has enabled the development of primary cell models and immunocompetent animal models based on easily obtainable 
laboratory animals. However, complementation of mouse and rat hepatocytes with hNTCP does not result in susceptibility to HBV infection (4-6), which indicates that additional host factors may be needed for the post-entry HBV lifecycle in mouse and rat hepatocytes. Therefore, other easily obtained laboratory animals that do not pose the same ethical concerns as primates may be a promising alternative solution for primary cell and animal models of HBV infection.

In the presence of $\mathrm{hNTCP}$, hepatocytes from primates are susceptible to HBV infection, whereas mouse and rat hepatocytes resist HBV infection (4-6). In the present study, it was presumed that animals with short evolutionary distance to primates may facilitate the HBV post-entry lifecycle. The generation of an easily obtained primary cell model for HBV infection based on other laboratory animals may be valuable for fundamental research on HBV infection and antiviral drugs. To achieve this goal, evolutionary distance was assessed based on single-copy homologous genes of seven species. The hepatocytes from candidate animals (pigs and rabbits) were then isolated, cultured and infected with hNTCP-recombinant lentivirus, followed by HBV infection. Anti-HBV drugs were also screened in the hNTCP-complemented hepatocytes.

\section{Materials and methods}

Cell isolation and culture. Ethical approval for the study was granted by the Institutional Bioethics Committee of Shenzhen Second People's Hospital, First Affiliated Hospital of Shenzhen University (Shenzhen, China). All animal experiments were performed in accordance with the Ministry of Health Guidelines for the Care and Use of Laboratory Animals (no. GB 14925-2001) and the ARRIVE guidelines.

Primary pig hepatocytes (PPHs) were isolated from the liver tissues of Wuzhishan minipigs $(n=3$; estimated age, 8 weeks) provided by the Beijing Genomics Institute, China. Primary rabbit hepatocytes (PRHs) were isolated from the liver tissues of New Zealand rabbits ( $n=4$; estimated age, 4 weeks) provided by Guangdong Medical Animal Center, China. Animals were euthanized by cutting the inferior vena cava under anaesthesia by intraperitoneal injection of $30 \mathrm{mg} / \mathrm{kg}$ pentobarbital sodium (7). Death was confirmed by respiratory arrest, cardiac arrest and reflex deficiency. The liver was removed as quickly as possible, perfused using a two-step collagenase perfusion procedure and plated as previously described $(8,9)$. Briefly, cell viability was $78-93 \%$, as determined by trypan blue staining and counting. Primary hepatocytes were seeded at a density of $2 \times 10^{5}$ viable cells $/ \mathrm{cm}^{2}$ in 12 -well tissue culture plates pre-coated with collagen (Gibco; Thermo Fisher Scientific, Inc.) in dimethyl sulfoxide (DMSO)-free primary human hepatocyte maintenance medium (PMM) (4) containing 5\% v/v fetal bovine serum (FBS; Gibco; Thermo Fisher Scientific, Inc.) for 4-6 h. Afterwards, the medium was replaced with fresh PMM. For the PRH culture, PMM containing 10\% $\mathrm{v} / \mathrm{v}$ FBS was essential during plating and maintenance. The cells were maintained at $37^{\circ} \mathrm{C}$ in a $5 \% \mathrm{CO}_{2}$ incubator, and the medium was replaced every 2 days.

Huh7D cells (10) were maintained in Dulbecco's modified Eagle's medium (DMEM; Gibco; Thermo Fisher Scientific, Inc.) supplemented with $10 \% \mathrm{FBS}, 100 \mathrm{U} / \mathrm{ml}$ penicillin and $100 \mu \mathrm{g} / \mathrm{ml}$ streptomycin maintained at $37^{\circ} \mathrm{C}$ in a $5 \% \mathrm{CO}_{2}$ incubator. Huh7D cells were induced using inducing medium (DMEM supplemented with 5\% FBS, $100 \mathrm{U} / \mathrm{ml}$ penicillin, $100 \mu \mathrm{g} / \mathrm{ml}$ streptomycin and $2.5 \%$ DMSO) for $24-72 \mathrm{~h}$ prior to HBV infection.

$H B V$ and lentivirus production. HepAD38 cells were kindly provided by Professor Chen Xulin (Wuhan Institute of Virology, CAS), with the permission of Professor Robert W. King (Institute for Cancer Research, Fox Chase Cancer Center, Philadelphia, PA, USA) (11). HepAD38 cells were maintained in Dulbecco's modified Eagle's medium (DMEM; Gibco; Thermo Fisher Scientific, Inc.) supplemented with 10\% FBS, $100 \mathrm{U} / \mathrm{ml}$ penicillin and $100 \mu \mathrm{g} / \mathrm{ml}$ streptomycin maintained at $37^{\circ} \mathrm{C}$ in a $5 \% \mathrm{CO}_{2}$ incubator. For the production of $\mathrm{HBV}$ particles, HepAD38 cells were maintained in Williams E medium supplemented with $10 \% \mathrm{FBS}, 100 \mathrm{U} / \mathrm{ml}$ penicillin and $100 \mu \mathrm{g} / \mathrm{ml}$ streptomycin, $5 \mu \mathrm{g} / \mathrm{ml}$ insulin, $18 \mathrm{ng} / \mathrm{ml}$ hydrocortisone and 2-2.5\% DMSO. The culture media was collected at an interval of 3-4 days. Concentrated HBV infectious particles were repaired as previously reported (12). Briefly, the supernatant was centrifuged to remove dead cells and debris at $1,000 \mathrm{xg}$ for $5 \mathrm{~min}$ at $4^{\circ} \mathrm{C}$. The supernatant was then filtered through a $0.45-\mu \mathrm{m}$ filter to remove any further debris. Subsequently, the supernatant was loaded into an ultrafiltration device with an intercepted molecular weight of $100 \mathrm{kDa}$ (EMD Millipore) and centrifuged at 1,000 x g for 30-60 min. The final volume for ultrafiltration was $250-500 \mu 1$, with a starting volume of $15 \mathrm{ml}$. The concentrated supernatant was collected and stored at $-80^{\circ} \mathrm{C}$. The hNTCP-recombinant lentivirus was produced as previously described (10). The concentrated lentivirus was stored at $-80^{\circ} \mathrm{C}$ until use in the experiments.

The pooled patient serum used for this study was reported elsewhere $(8,9)$, of which personal permissions were obtained from $\mathrm{HBV}$-infected patients. HBV particles purified from patient serum were described previously (12). Briefly, Nycodenz (Takeda Pharmaceutical Company, Ltd.) was dissolved in Hepato-STIM Hepatocyte Defined Medium (BD Biosciences). Serum from patients with HBV (viral load $>10^{8}$ copies $/ \mathrm{ml}$ ) was loaded onto the Nycodenz gradient (range, $8-50 \%$ ) in $11 \times 34 \mathrm{~mm}$ polycarbonate centrifuge tubes (Beckman Coulter, Inc.). The samples were centrifuged in a TLS55 swing-out rotor (Beckman Coulter, Inc.) for $45 \mathrm{~min}$ at $200,000 \mathrm{x}$ g at $20^{\circ} \mathrm{C}$. Subsequently, $155 \mu \mathrm{l}$ aliquots were consecutively removed (fractions 1 to 8). Fractions 6 and 7 were pooled with $8.4 \times 10^{8}$ $\mathrm{HBV}$ copies/ml, as measured by quantitative PCR (qPCR).

HBV infection. PPHs and PRHs were seeded and maintained at $100 \%$ confluence. Huh7D cells (10) were seeded at $50 \%$ confluence and cultured to $80 \%$ confluence prior to induction with $2.5 \%$ DMSO. Cells were infected with hNTCP-recombinant lentivirus in the presence of $6 \mu \mathrm{g} / \mathrm{ml}$ polybrene (Sigma-Aldrich; Merck KGaA). The medium was replaced at $12 \mathrm{~h}$. To ensure expression of hNTCP protein, cells were maintained for at least $72 \mathrm{~h}$ prior to HBV infection. At 3 days post-lentivirus infection, concentrated HBV stock solution diluted in PMM (10\% v/v FBS was added for PRHs) supplemented with 4\% (w/v) PEG8000 was added directly to the cells with a multiplicity of infection (MOI) of 1,000 . For HBV infection with purified patient serum, a MOI of 200 was used. Following incubation overnight, cells were washed 
thoroughly three times with phosphate-buffered saline, and the media were refreshed. Cells were maintained at $37^{\circ} \mathrm{C}$ in a $5 \% \mathrm{CO}_{2}$ incubator, and the medium was changed every 2 days.

For the competing infection assays, the cells were pre-incubated with different concentrations of the HBV entry inhibitor myr-preS $1^{2-47}$ at $37^{\circ} \mathrm{C}$ for $30 \mathrm{~min}$ in a $5 \% \mathrm{CO}_{2}$ incubator, and infectious medium containing myr-preS1 ${ }^{2-47}$ was added. For the blocking infection assays, infectious media were pre-incubated with different concentrations of the HBV entry inhibitor 4B10 at $37^{\circ} \mathrm{C}$ for $30 \mathrm{~min}$ in a $5 \% \mathrm{CO}_{2}$ incubator, and the infectious medium (containing 4B10) was added to the cells. For the inhibition of $\mathrm{HBV}$ replication assays, different concentrations of Lamivudine were incubated with cells post-HBV infection.

Cytotoxicity assay. For cytotoxicity of patients' pool serum, the pool serum was incubated with hNTCP-expressing PPH or heat-inactivated at $56^{\circ} \mathrm{C}$ for $30 \mathrm{~min}$ before incubation. Cell viabilities were measured by Cell Counting Kit- 8 from Yeasen Biotechnology (Shanghai) Co., Ltd., according to the manufacturer's instructions. For cytotoxicity of inhibitors, different concentrations of inhibitors (myr-preS1 ${ }^{2-47}, 4 \mathrm{~B} 10$ and Lamivudine) were incubated with hNTCP-expressing PPH for 48 h. Cell viabilities were measured by Cell Counting Kit- 8 according to the manufacturer's instructions.

ELISA. Secreted hepatitis B surface antigen (HBsAg) and hepatitis $\mathrm{B}$ e-antigen ( $\mathrm{HBeAg}$ ) were detected in medium collected from $\mathrm{HBV}$-infected cells using a $\mathrm{HBsAg} / \mathrm{HBeAg}$ ELISA kit (Shanghai Kehua Bio-engineering Co., Ltd), according to the manufacturer's instructions. The results were assessed using a Multiskan MK3 microplate spectrophotometer (Thermo Fisher Scientific, Inc.). The optical density (OD) values are presented as the mean \pm standard deviation of $\mathrm{OD}_{450}-\mathrm{OD}_{630}$. The cut-off value was calculated according to the manufacturer's instructions.

PCR analysis. HBV covalently closed circular DNA (cccDNA) was extracted as described by Köck et al (13). Briefly, $\sim 2 \times 10^{6}$ cells in a single well in a 6 -well culture plate were lysed with $500 \mu \mathrm{l}$ NP-40 lysis buffer (50 mM Tris pH 8.0, $140 \mathrm{mM}$ $\mathrm{NaCl}$ and $0.5 \% \mathrm{NP}-40$ ) for 5-10 min until the cells detached from the well. Cells were thoroughly rinsed several times with the NP-40 lysis buffer. The lysate (nuclei and cell debris) was centrifuged for $5 \mathrm{~min}$ at $1,500 \mathrm{x} \mathrm{g}$, and the supernatant was removed. A total of $500 \mu 1 \mathrm{NP}-40$ lysis buffer, $5 \mu 1$ ethylenediaminetetraacetic acid (EDTA; $0.5 \mathrm{M}$ ) and $5 \mu$ proteinase $\mathrm{K}$ (20 mg/ml; Tiangen Biotech Co., Ltd.) were added to the nuclear pellet and incubated at $56^{\circ} \mathrm{C}$ for $2 \mathrm{~h}$. The mixture was loaded onto a QIAshredder column (Qiagen $\mathrm{GmbH}$ ) and centrifuged at room temperature for $1 \mathrm{~min}$ at $15,800 \mathrm{x} \mathrm{g}$. The flow-through was incubated at $56^{\circ} \mathrm{C}$ for $1 \mathrm{~h}$, the DNA was purified by phenol-chloroform extraction and ethanol precipitation, and the pellet was solubilized in $\mathrm{ddH}_{2} \mathrm{O}$. The genomic DNA, linear viral DNA and relaxed circular DNA were digested for $8 \mathrm{~h}$ at $37^{\circ} \mathrm{C}$ by plasmid-safe ATP-dependent deoxyribonuclease DNase (Epicentre; Illumina, Inc.). Following inactivation at $70^{\circ} \mathrm{C}$ for $30 \mathrm{~min}$, cccDNA was detected with the primers ccc-1580 and ccc-2314 (Table I) (4,14). A 10-fold dilution series of plasmid pGEM-3Z-1.3xHBV (15) was used as the standard.
The hNTCP mRNA level was detected as described previously (9). Briefly, total intracellular RNA was extracted using TRIzol ${ }^{\circledR}$ reagent (Invitrogen; Thermo Fisher Scientific, Inc.), and cDNA was synthesized using the PrimeScript RT kit with gDNA Eraser (Takara Biotechnology Co., Ltd.). The cDNA products were subjected to GPCR with primers for hNTCP and $\beta$-actin (Table I). $\beta$-actin served as the internal control for sample normalization. The relative hNTCP mRNA expression levels were calculated using the $2^{-\Delta \Delta \mathrm{Cq}}$ method (16) and the expression of each group was calculated as relative to the levels of Huh7D cells. All samples were measured in triplicate and all experiments were repeated independently three times.

For quantification of HBV copies, HBV DNA was extracted using QIAamp DNA Blood Mini kit (Qiagen $\mathrm{GmbH}$ ), according to the manufacturer's instructions. qPCR was performed using FastStart Universal SYBR ${ }^{\circledR}$ Green Master Mix (Roche Diagnostics). A pCH9-3091 (17) plasmid (containing 1.05X HBV genomic DNA) was used as the standard. The exact HBV copies were calculated with molecular weight of plasmid, plasmid concentration and Avogadro constant. The primer sequences and the thermocycling conditions for all qPCR experiments are presented in Table I.

Western blotting. Western blotting was performed as previously described (8). The primary antibodies used were rabbit anti-human hNTCP polyclonal antibody (cat. no. HPA042727; Sigma-Aldrich; Merck KGaA; dilution 1:1,000), and mouse anti-human $\beta$-actin monoclonal antibody (cat. no. 60008-1-Ig Proteintech Group, Inc.; dilution 1:1,000). The secondary antibodies used were HRP-conjugated Affinipure Goat Anti-Rabbit IgG(H+L) (cat. no. SA00001-2; Proteintech Group, Inc.; dilution 1:5,000), HRP-conjugated Affinipure Goat Anti-Mouse IgG(H+L) (cat. no. SA00001-1; Proteintech Group, Inc.; dilution 1:5,000).

Immunofluorescence assay. Immunofluorescence assay was performed as described previously (8). The primary antibody used was anti-rhodopsin antibody (cat. no. ab5417; Abcam; dilution 1:1,000) rabbit anti-human $\mathrm{hNTCP}$ polyclonal antibody (cat. no. HPA042727; Sigma-Aldrich; Merck KGaA; dilution 1:750). The secondary antibody used was Alexa Fluor Plus 488-conjugated Goat anti-Rabbit IgG (H+L) (cat. no. A32731; Thermo Fisher Scientific, Inc.; dilution 1:1,000). The nuclei were stained with 4',6-diamidino-2-phenylindole (DAPI; Roche Diagnostics; dilution 1:2,000), and the cells were examined under a fluorescence microscope (Leica Microsystems $\mathrm{GmbH}$ ), magnification, $\mathrm{x} 200$. The images were processed using Adobe Photoshop CS5 (Adobe Systems, Inc.).

Divergence tree. A divergence tree was created based on the single-copy homologous genes of the following species: Homo sapiens (human), Macaca mulatta (rhesus macaque), Sus scrofa (domestic pig), Tadarida brasiliensis (bat), Oryctolagus cuniculus (rabbit), Mus musculus (mouse) and Rattus norvegicus (rat) using the OrthoMCL v2.0 (http://orthomcl.org/orthomcl/), Muscle (https://www.ebi.ac.uk/Tools/msa/muscle/), PHYML v3.0 (http://www.atgc-montpellier.fr/phyml/), and MCMCTree v4.4 (http://abacus.gene.ucl.ac.uk/software/paml.html) software. 
Table I. Primers used for PCR.

\begin{tabular}{lll}
\hline Primer & \multicolumn{1}{c}{ Sequence (5'-3') } & \multicolumn{1}{c}{ Thermocycling conditions } \\
\hline $\operatorname{ccc} 1580$ & F: TGCACTTCGCTTCACCT & 45 cycles: $95^{\circ} \mathrm{C}$ for $10 \mathrm{sec}, 61^{\circ} \mathrm{C}$ for $20 \mathrm{sec}, 72^{\circ} \mathrm{C}$ for $40 \mathrm{sec}$ \\
$\operatorname{ccc} 2314$ & R: AGGGGCATTTGGTGGTC & \\
hNTCP & F: CTCAAATCCAAACGGCCACAATAC & 40 cycles: $95^{\circ} \mathrm{C}$ for $30 \mathrm{sec}, 60^{\circ} \mathrm{C}$ for $20 \mathrm{sec}, 72^{\circ} \mathrm{C}$ for $30 \mathrm{sec}$ \\
& R: CACACTGCACAAGAGAATGATGATC & \\
HBVp & F: ACCAATCGCCAGTCAGGAAG & 40 cycles: $95^{\circ} \mathrm{C}$ for $30 \mathrm{sec}, 60^{\circ} \mathrm{C}$ for $30 \mathrm{sec}, 72^{\circ} \mathrm{C}$ for $30 \mathrm{sec}$ \\
& R: ACCAGCAGGGAAATACAGGC & \\
$\beta$-actin & F: ATCGTGCGTGACATTAAGGAG & 40 cycles: $95^{\circ} \mathrm{C}$ for $30 \mathrm{sec}, 60^{\circ} \mathrm{C}$ for $20 \mathrm{sec}, 72^{\circ} \mathrm{C}$ for $30 \mathrm{sec}$ \\
& R: GGAAGGAAGGCTGGAAGAGT &
\end{tabular}

F, forward; R, reverse; hNTCP, human sodium taurocholate co-transporting polypeptide; HBVp, primers for HBV copies locates in the encoding sequence of polymerase.

Statistical analysis. The results are presented as the mean \pm SD. Statistical analysis was performed on GraphPad Prism 6 (GraphPad Software, Inc.) using Student's t-test or one-way ANOVA followed by Least Significant Difference post hoc test. $\mathrm{P}<0.05$ was considered to indicate a statistically significant difference.

\section{Results}

Evolutionary distance based on single-copy homologous genes. The use of animals close to $H$. sapiens and M. mulatta in the divergence tree for the isolation of primary hepatocytes may facilitate the HBV post-entry lifecycle. Based on the single-copy homologous genes of seven species (18), a divergence tree was generated (Fig. 1). The divergence years were estimated to be in the following order: M. musculus and $R$. norvegicus (84.9 million years) $>$ O. cuniculus (69.8 million years) $>H$. sapiens and M. mulatta (59.9 million years) > S. scrofa and T. brasiliensis. Primary hepatocytes isolated from $H$. sapiens and $M$. mulatta fully support the HBV post-entry lifecycle, whereas hepatocytes from $M$. musculus and $R$. norvegicus are resistant (4-6). In addition, bats, such as $T$. brasiliensis, carry pathogenic hepadnaviruses that are antigenically related to HBV and are capable of infecting human hepatocytes (19), indicating that T. brasiliensis hepatocytes may also facilitate the HBV post-entry lifecycle as hepadnaviruses are highly host selective. Notably, hepadnavirus has been detected in $S$. scrofa (domestic pigs) $(20,21)$, and $O$. cuniculus (rabbits), which may also be infected by an HBV-like virus (22). Therefore, easily obtained laboratory animals, such as pigs and rabbits, may be suitable as potential hosts for HBV infection after hNTCP complementation.

Culture and hNTCP complementation of PPHs and PRHs. PPHs and PRHs were isolated by a two-step collagenase digestion and plated on collagen-coated culture plates. PPHs were maintained in PMM (4) for $>2$ weeks and exhibited a typical highly differentiated morphology, such as spherical bright nuclei and a polarized shape (Fig. 2A). However, PRHs rapidly deteriorated in PMM and died within 2 days (data not shown). Following optimization, PRHs could be maintained in PMM supplemented with $10 \%$ FBS for approximately 10-14 days and

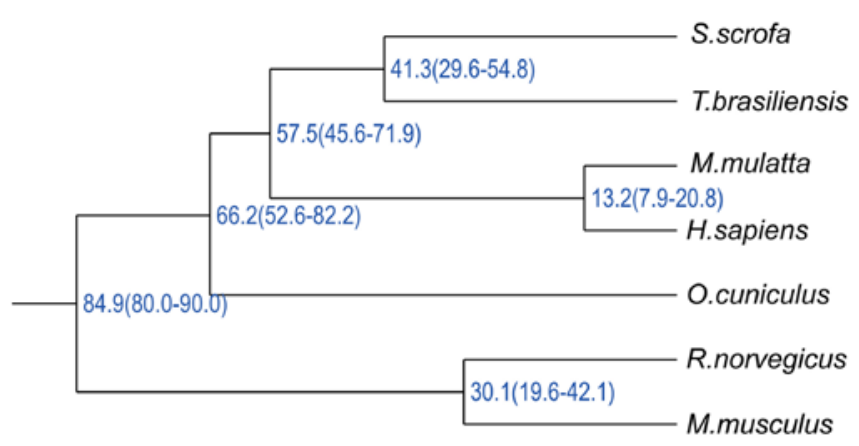

$180, ', 160, \quad, 140,1,1201,101$ Million years ago

Figure 1. Evolutionary distance based on single-copy homologous genes. A divergence tree was generated based on single-copy homologous genes of seven species using Orthomcl, Muscle, PHYML v3.0 and Mcmctree v4.4 software.

exhibited a typical highly differentiated morphology (Fig. 2A). The difference in maintenance medium between PPHs and PRHs indicated that PPHs may more closely resemble PHHs compared with PRHs, which matched the evolutionary distances identified in the divergence tree (Fig. 1).

As a positive control for HBV infection, the human cell line Huh7D exhibits increased susceptibility to HBV infection in the presence of hNTCP compared with the HepG 2 cell line (10); therefore, the Huh7D cell line was selected in the present study as a positive control for subsequent experiments. PPHs and PRHs, as well as Huh7D cells, were infected with the hNTCP-recombinant lentivirus. Cells were harvested 4 days post-lentiviral infection to determine the hNTCP mRNA expression levels by qPCR and protein expression levels by western blotting, or were fixed to detect hNTCP expression and location by immunofluorescence assay. hNTCP expression was only observed in cells infected with hNTCP-recombinant lentivirus (Fig. 2B and C). Although the same MOI of the lentivirus $(\mathrm{MOI}=1)$ was used, the estimated percentage of hNTCP-positive PPHs and Huh7D cells was 70-80\%, whereas the estimated percentage of hNTCP-positive PRHs was slightly lower at 50-60\% (Fig. 2D). These hNTCP-expressing cells were subsequently subjected to HBV infection. 

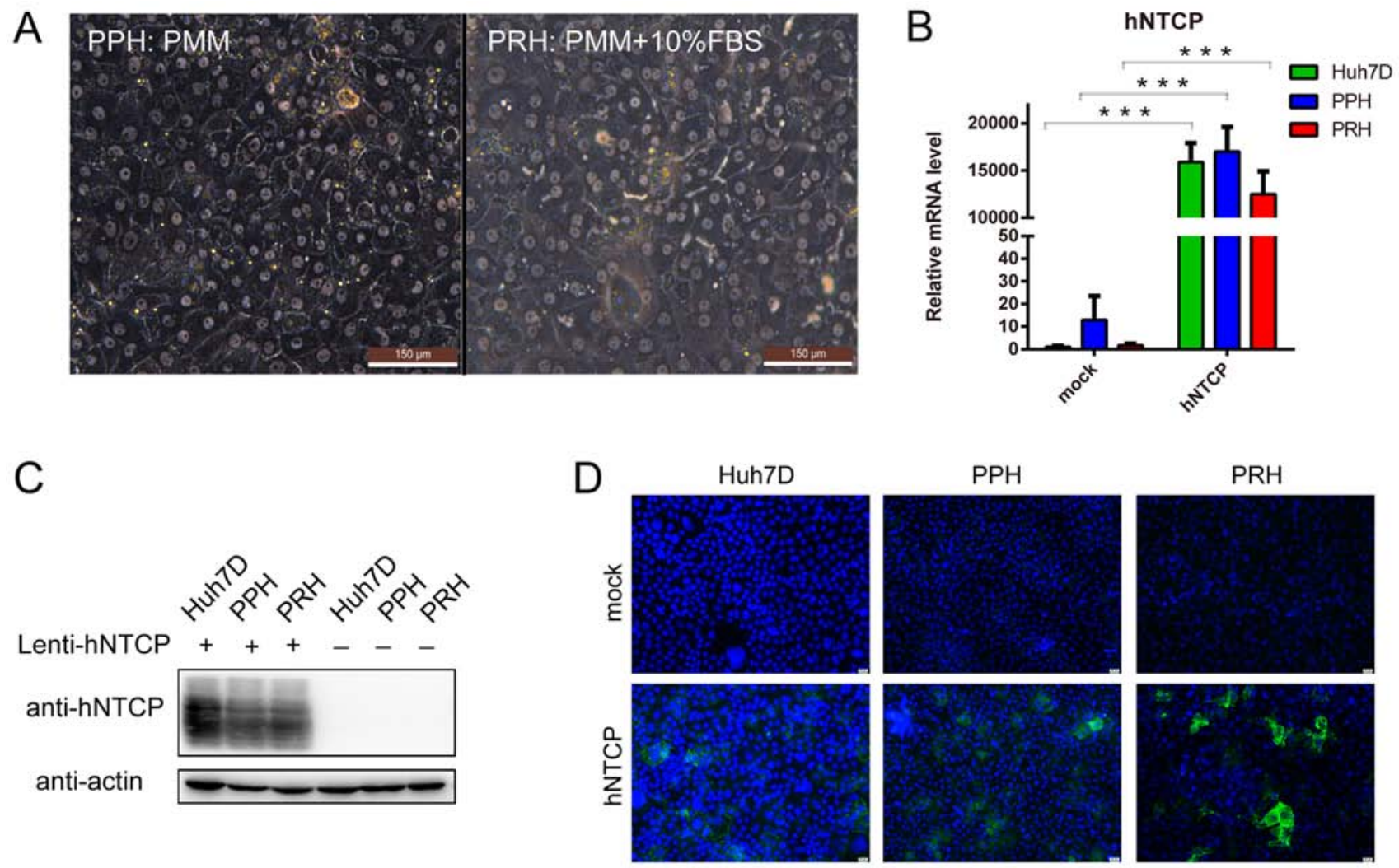

Figure 2. Culture and hNTCP complementation of PPHs and PRHs. (A) The morphology of PPHs and PRHs was assessed at 4 days post-seeding using a phase-contrast microscope. Scale bar, $150 \mu \mathrm{m}$. The maintenance medium is indicated. (B) The different cell groups were subjected to reverse transcription-quantitative PCR to determine the relative hNTCP mRNA expression levels. (C) Lysates of the different cell groups were subjected to western blotting to detect hNTCP protein expression levels. (D) At 4 days post-lentivirus infection, the different cell types groups were analyzed by immunofluorescence. Green, hNTCP staining; blue, nucleus. Scale bar, $25 \mu \mathrm{m} .{ }^{* * * *} \mathrm{P}<0.001$, with comparisons indicated by lines. hNTCP, human sodium taurocholate co-transporting polypeptide; PPH, primary pig hepatocyte; $\mathrm{PRH}$, primary rabbit hepatocyte; PMM, primary human hepatocyte maintenance medium; FBS, fetal bovine serum.

$H B V$ infection of primary hepatocytes. HBV infection was performed using concentrated viral stocks prepared from HepAD38 cells (20). According to our preliminary data, the hNTCP-complemented PPHs could be maintained for $>2$ weeks post-HBV infection, whereas hNTCP-complemented PRHs could be maintained for 1 week (data not shown). Therefore, the experimental period was limited to 7 days post-infection (dpi). Following infection with HBV (MOI=1,000), all cell types without hNTCP exhibited either negative or reduced secretion of $\mathrm{HBsAg} / \mathrm{HBeAg}$, indicating that these cells were resistant to HBV infection. Following hNTCP complementation, Huh7D and PPHs, but not PRHs, were positive for $\mathrm{HBs} \mathrm{Ag} / \mathrm{HBeAg}$ secretion, with continuously increased secretion of $\mathrm{HBsAg}$ and $\mathrm{HBeAg}$ until the end of culture (Fig. 3A and B). Of note, HBsAg and HBeAg secretion in hNTCP-complemented PPHs was significantly higher compared with the levels in hNTCP-complemented Huh7D cells, although hNTCP mRNA and protein levels were similar in these cells (Fig. 2B and C).

To confirm the formation of cccDNA, the infected cells were lysed, and nuclear DNA was extracted for qPCR. As presented in Fig. 3C, hNTCP-complemented PPHs produced the highest cccDNA levels among the three types of cells, followed by hNTCP-complemented Huh7D cells, whereas the cccDNA levels in hNTCP-complemented PRHs were comparable to the controls. These results were similar with the results of $\mathrm{HBeAg}$ and $\mathrm{HBsAg}$ secretion. To further confirm the secretion of infectious HBV particles, the new virions in the culture medium at 3,5, and $7 \mathrm{dpi}$ were concentrated by PEG8000 and added to a new batch of hNTCP-complemented PPHs (Fig. 3D). HBeAg secretion in infected hNTCP-complemented PPHs gradually increased in a time-dependent manner, indicating that new virions were infectious and could be passaged (Fig. 3D).

Serum from HBV-positive patients is frequently used as a viral source for infection, as it is related to clinical viral strains. hNTCP-complemented PPHs were infected at $\mathrm{MOI}=200$ with raw pooled patient serum and purified HBV particles. As presented in Fig. 3E, HBeAg secretion was positive and continuously increased during HBV infection with purified HBV particles, indicating that hNTCP-complemented PPHs could be infected by clinical viral strains. Raw pooled patient serum was cytotoxic to PPHs, as the cells died in the presence of this serum (data not shown). This cytotoxicity was significantly reduced by heating the raw pooled patient serum at $56^{\circ} \mathrm{C}$ for $30 \mathrm{~min}$ (Fig. 3F), indicating that natural human anti-pig antibodies (such as anti-Gal or anti-Neu5Gc antibodies) and the complement system were the major cause of cytotoxicity. Therefore, purification is essential for the establishment of HBV infection using human serum as the viral source.

Anti-HBV drugs may be screened in the hNTCPcomplemented PPHs. Lamivudine, Myr-preS1 ${ }^{2-47}$ and 
A

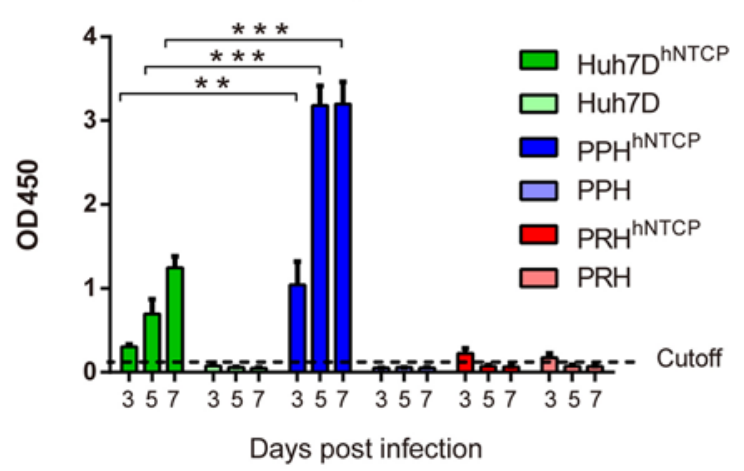

C

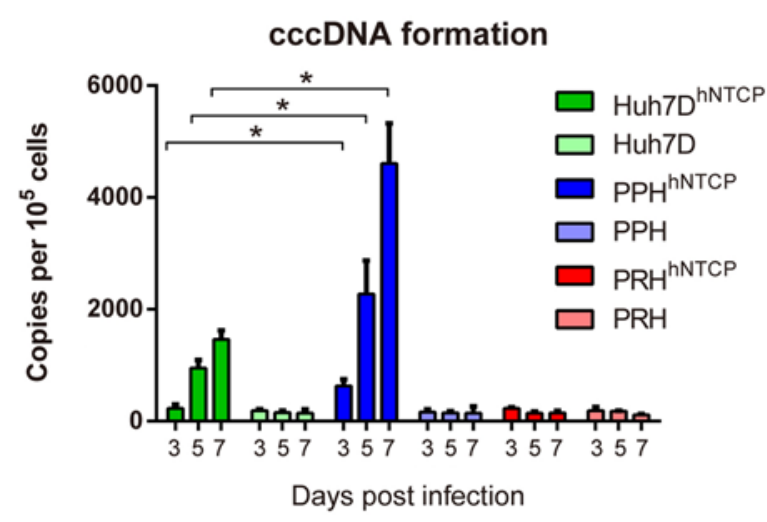

E

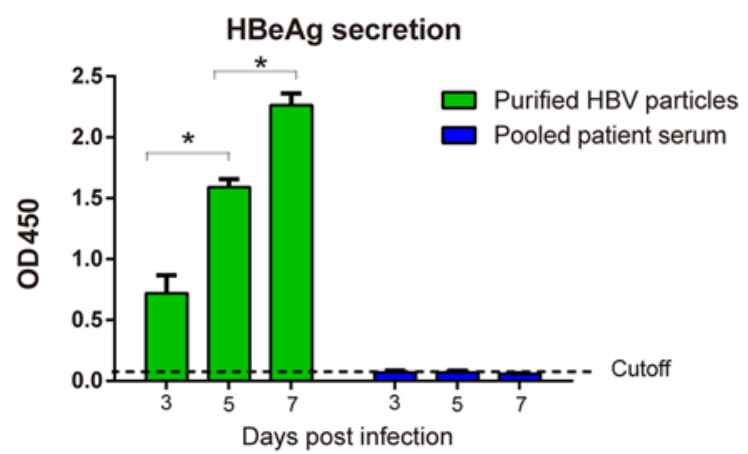

B

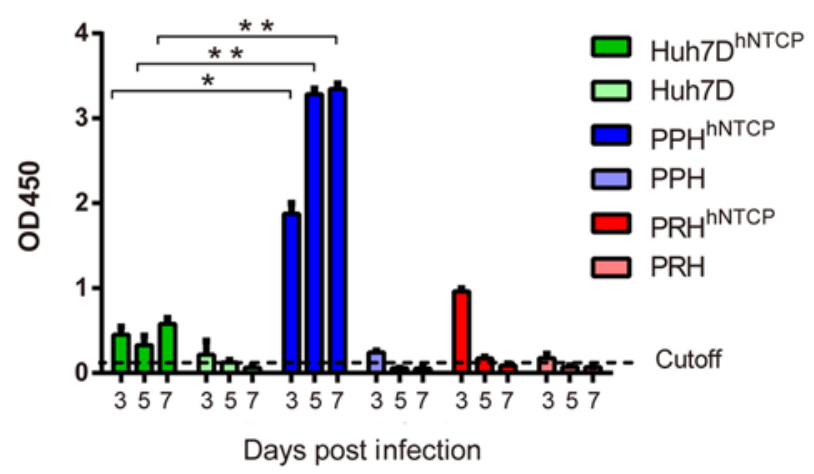

D

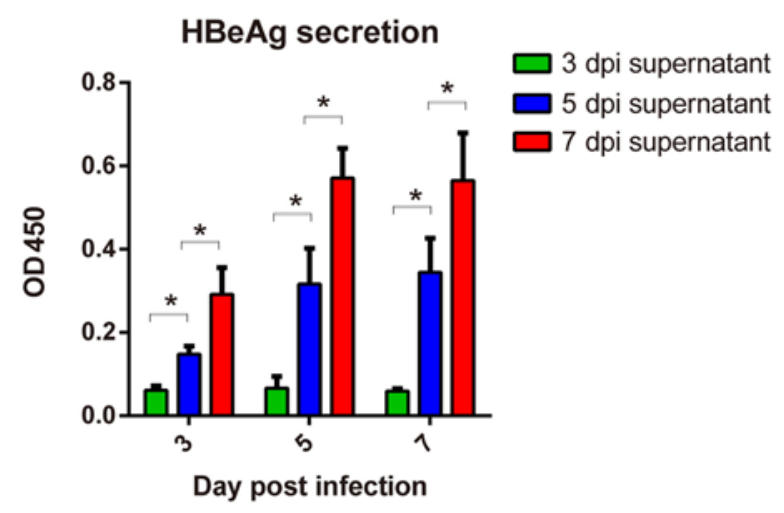

F

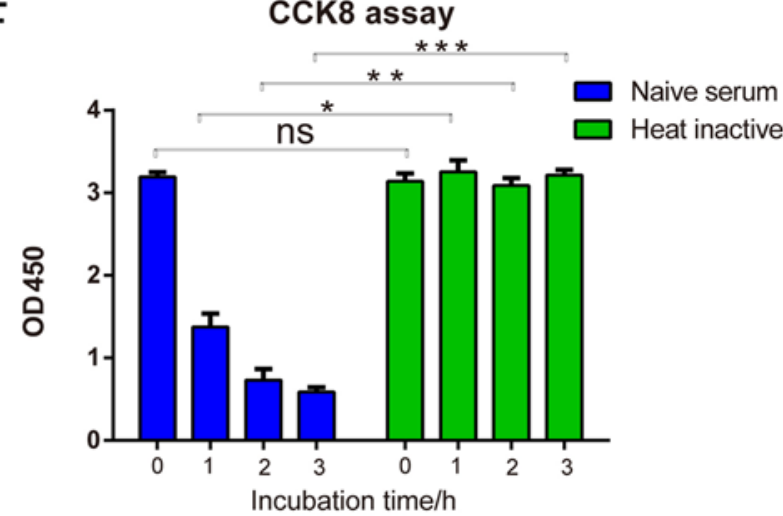

Figure 3. HBV infection of hNTCP-complemented hepatocytes. (A-C) Cells were infected with HBV stocks for 16-24 h, and media were collected at 3, 5 and 7 dpi for detection of (A) HBeAg and (B) HBsAg by ELISA, and (C) cccDNA by quantitative PCR. Values over the cut-off line were considered positive. (D) HBV infections were performed using PEG8000-concentrated supernatants at 3, 5 and 7 dpi, and HBeAg secretion was measured by ELISA at the indicated time points. (E) Raw HBV-positive serum and purified HBV particles served as viral sources to infect hNTCP-complemented PPHs, followed by HBeAg detection. (F) hNTCP-complemented PPHs were incubated for the indicated time points with raw patient serum (naïve serum) and heated patient serum, and cell viabilities were detected by Cell Counting Kit- $8 .{ }^{*} \mathrm{P}<0.05,{ }^{* *} \mathrm{P}<0.01$ and ${ }^{* * * *} \mathrm{P}<0.001$, with comparisons indicated by lines. hNTCP, human sodium taurocholate co-transporting polypeptide; $\mathrm{PPH}$, primary pig hepatocyte; $\mathrm{PRH}$, primary rabbit hepatocyte; HBV, hepatitis $\mathrm{B}$ virus; dpi, days post-infection; HBsAg, hepatitis B surface antigen; HBeAG, hepatitis B e-antigen; ns, not significant.

4B10 are well-characterized inhibitors of HBV replication and/or HBV entry (23-25) and were tested in HBV-infected hNTCP-complemented PPHs in the present study. The three inhibitors were non-cytotoxic to hNTCP-complemented PPHs (Fig. 4D). Myr-preS1 ${ }^{2-47}$ and 4B10, the peptide HBV entry inhibitors, exhibited concentration-dependent inhibition of HBsAg and HBeAg secretion and HBV DNA in the supernatants as higher concentration of inhibitors significantly reduced antigens/virion secretion compared with the lower concentration although there is a plateau, with a half maximal inhibitory concentration $\left(\mathrm{IC}_{50}\right)$ of $0.5-1 \mathrm{nM}$ (Fig. 4A-C), which matches the reported data regarding $\mathrm{HBV}$ infection in the $\mathrm{PHH}$ model (23). Lamivudine, an inhibitor of HBV replication, also exhibited concentration-dependent inhibition of HBsAg and HBeAg secretion and HBV DNA in the supernatants, with an $\mathrm{IC}_{50}$ of $\sim 50 \mathrm{nM}$. The less effective inhibition of HBV infection by lamivudine possibly occurred due to the failure of lamivudine to block HBV entry. These results indicated that 
A

HBsAg secretion

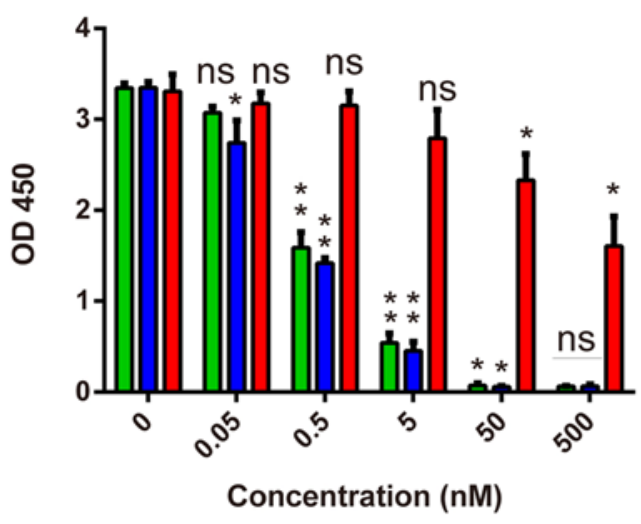

C HBV DNA in supernatants

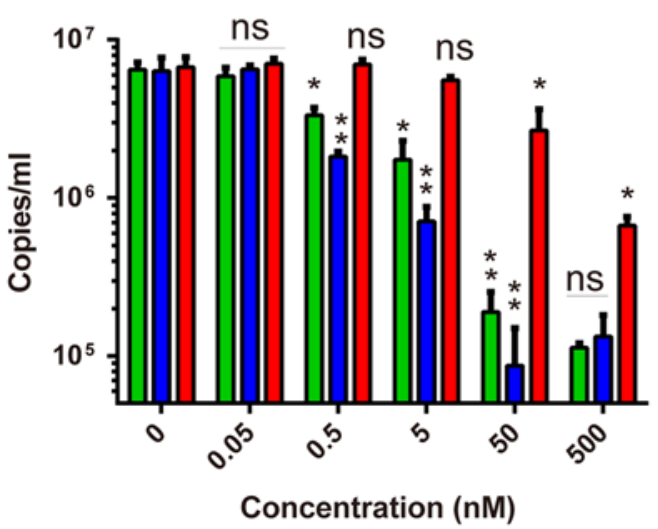

B HBeAg secretion
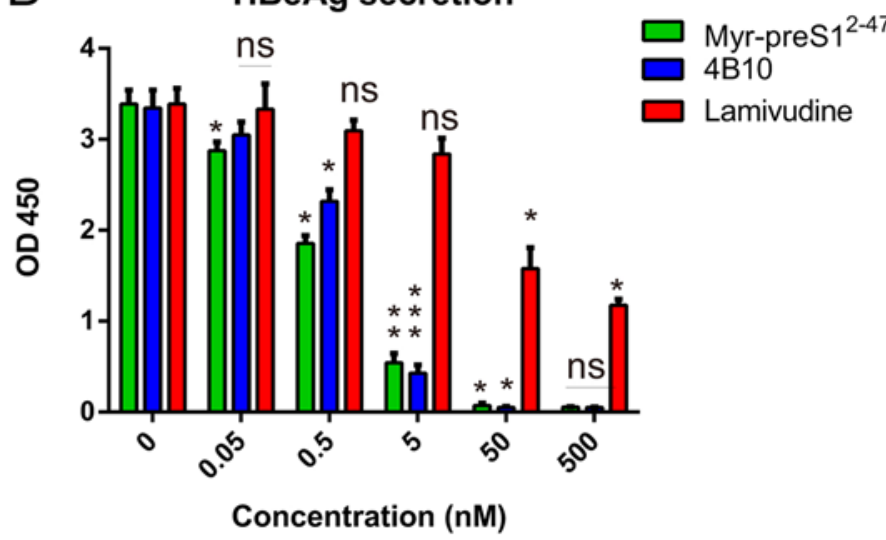

$\mathrm{D}$

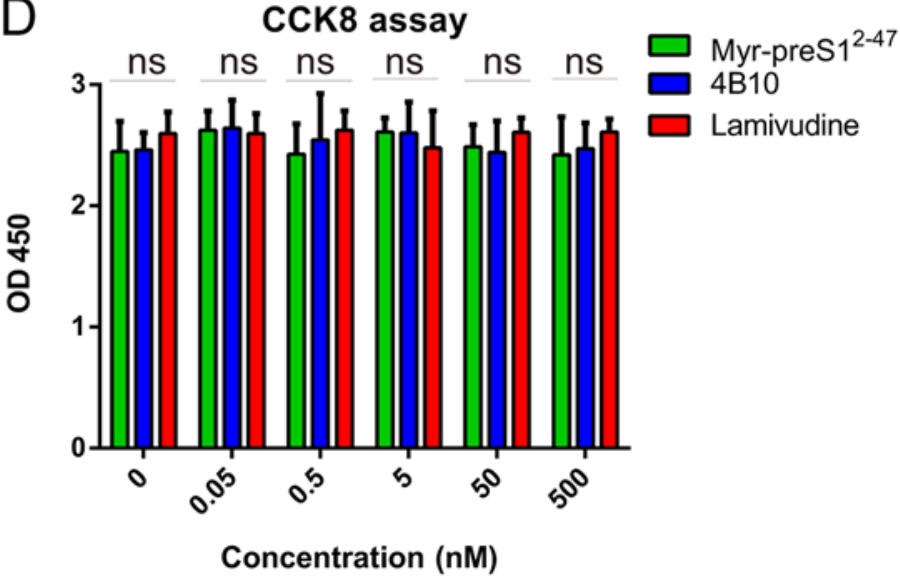

Figure 4. Anti-HBV drugs tested in HBV-infected hNTCP-complemented primary PPHs. HBV infections of HepAD38 cells were performed in the presence of different doses of Lamivudine, Myr-preS12-47 and 4B10. (A) Relative HBsAg and (B) HBeAg secretion in the culture supernatant 7 days post-infection was measured by ELISA. (C) HBV copies in the supernatant were measured by quantitative PCR. (D) Lamivudine, Myr-preS1 ${ }^{2-47}$ and $4 \mathrm{~B} 10$ were not cytotoxic to hNTCP-complemented PPHs, as evidenced by Cell Counting Kit- 8 assay. ${ }^{*} \mathrm{P}<0.05,{ }^{* *} \mathrm{P}<0.01$ and ${ }^{* * * *} \mathrm{P}<0.001$, with comparisons indicated by lines. hNTCP, human sodium taurocholate co-transporting polypeptide; $\mathrm{PPH}$, primary pig hepatocyte; HBV, hepatitis B virus; HBsAg, hepatitis B surface antigen; HBeAg, hepatitis B e-antigen; ns, not significant.

HBV-infected hNTCP-complemented PPHs may be applicable for anti-HBV drug screening.

\section{Discussion}

The results of the present study indicated species tropism regarding the HBV post-entry lifecycle. S. scrofa was hypothesized to facilitate the HBV post-entry lifecycle, as it is close to $T$. brasiliensis in the divergence tree, both of which are close to $H$. sapiens and M. mulatta. S. scrofa was experimentally demonstrated to facilitate the HBV post-entry lifecycle in the present study. Although $O$. cuniculus is closer to H. sapiens and M. mulatta compared with M. musculus and R. norvegicus, PRHs were unable to facilitate the HBV post-entry lifecycle in the present study. According to the present results, $R$. norvegicus and O. cuniculus may lack one or more limiting factors for the HBV post-entry lifecycle. Identification of these limiting factors may help in the development of immunocompetent small animal models and identification of novel antiviral targets.

As demonstrated in the present study, the differentiation status is an important limiting factor in productive $\mathrm{HBV}$ infection and replication. According to viral antigen secretion and cccDNA formation, hNTCP-complemented PPHs may be a superior cell model for HBV infection compared with the dedifferentiated human hepatoma cell line Huh7D (10). The Huh7 cell line can be induced by DMSO to a higher differentiation state compared with untreated cells. Our previous study has also demonstrated this phenomenon (10). However, the differentiation state of well-differentiated Huh7 is still lower compared with that of primary human hepatocytes. For example, well-differentiated Huh7 is resistant to HBV infection. According to our previous unpublished data, the expression levels of differentiation-related genes are lower in well-differentiated Huh7 cells compared with primary human hepatocyte. As pig is close to human in the divergence tree, PPHs complemented with hNTCP may be a superior model for HBV infection compared with the dedifferentiated human hepatoma cell line.

hNTCP expression in PPHs is controlled by a relatively stable promoter (human elongation factor-1 $\alpha$ ); this is different from the endogenous promoter that induces hNTCP expression in PHHs, which leads to a rapid decrease in hNTCP expression, thus causing PHHs to be resistant to HBV infection (9). Therefore, hNTCP-complemented PPHs may be more susceptible to HBV infection compared with PHHs. 
However, hNTCP-complemented PPHs have certain limitations, such as genomic differences compared with PHHs and limited genomic annotation. One of these differences is the xenoantigens expressed on pig cells (e.g., Gal and Neu5Gc), which can be recognized by natural anti-pig antibodies in human blood and may lead to complement-dependent cytotoxicity (26). This may explain why hNTCP-complemented PPHs were fragile when raw patient serum was used as the viral source in the present study. However, hNTCP-complemented PPHs may partially replace the roles of PHHs in HBV-related fundamental research and drug screening.

The MOI for HBV infection of PPHs using HepAD38 cells as viral source was different compared with that using patient serum as viral source. The majority of viral particles from HepaAD38 are naked, whereas the majority of viral particles in the patient serum are intact. Therefore, different MOI was used in HBV infection with different viral sources, which has been suggested for successful HBV infection in our previous publications (8-10).

Lempp et al (27) have reported that hNTCP is the limiting factor in HBV infection in pig hepatocytes, which was also demonstrated in the present study. In addition, the possibility of $O$. cuniculus facilitating the HBV post-entry lifecycle was assessed and subsequently excluded from the present study, which supplemented the literature on potential animal hosts for HBV infection.

In conclusion, hNTCP-complemented PPHs may be a valuable tool for investigating HBV infection and antiviral drugs. The results also indicated that the pig is a promising candidate in the development of an immunocompetent animal model.

\section{Acknowledgements}

The authors would like to thank Professor Chen Xulin from Wuhan Institute of Virology, CAS (Wuhan) for kindly providing HepAD38. They are also grateful to Professor M Nassal (University of Freiburg) for kindly providing the protocol of cccDNA extraction.

\section{Funding}

This work was supported by the National Natural Science Foundation of China (grant. no. 81601760), the General Financial Grant from the China Postdoctoral Science Foundation (grant. no. 2016M602587), the Shenzhen Foundation of Science and Technology (grant. no. JCYJ20 160425104534335 to MZ), the Health and Family Planning Commission of Shaoxing, Zhejiang Province, China (grant. no. 2017QN001), the Science Technology Bureau of Shaoxing, Zhejiang Province, China (grant. no. 2017B70022 to BQ), the Sanming Project of Medicine in Shenzhen (grant. no. SZSM201412020 to LSM) and Special Funds for the Construction of High Level Hospitals in Guangdong Province (2019).

\section{Availability of data and materials}

The datasets generated and/or analysed during the current study are available from the corresponding authors on reasonable request.

\section{Authors' contributions}

LSM and MZ conceived and designed the study. MZ and BQ completed data acquisition, analysis and interpretation in this study. MZ wrote the manuscript. XSD, XLZ, BQ, YL, ZGH and $\mathrm{CCW}$ performed the experiments. $\mathrm{CCW}$ reviewed and edited the manuscript. All authors have read and approved the final manuscript.

\section{Ethics approval and consent to participate}

This study was approved by the Institutional Bioethics Committee of Shenzhen Second People's Hospital, First Affiliated Hospital of Shenzhen University, Shenzhen, China. All animal experiments were performed in accordance with the Ministry of Health Guidelines for the Care and Use of Laboratory Animals (no. GB 14925-2001), and all procedures were approved by the Laboratory Animal Ethics Committee of the First Affiliated Hospital of Shenzhen University. All experiments were performed in accordance with the relevant guidelines and regulations. The manuscript was prepared and revised according to the ARRIVE guidelines.

\section{Patient consent for publication}

Not applicable.

\section{Competing interests}

The authors declare that they have no competing interests.

\section{References}

1. Beasley RP: Rocks along the road to the control of HBV and HCC. Ann Epidemiol 19: 231-234, 2009.

2. Sheahan T, Jones CT and Ploss A: Advances and challenges in studying hepatitis $C$ virus in its native environment. Expert Rev Gastroenterol Hepatol 4: 541-550, 2010.

3. Weizsäcker FV and Roggendorf M: Models of viral hepatitis. Basel; New York, Karger, 2005.

4. Yan H, Zhong G, Xu G, He W, Jing Z, Gao Z, Huang Y, Qi Y, Peng B, Wang $\mathrm{H}$, et al: Sodium taurocholate cotransporting polypeptide is a functional receptor for human hepatitis B and D virus. Elife 1: e00049, 2012.

5. He W, Ren B, Mao F, Jing Z, Li Y, Liu Y, Peng B, Yan H, Qi Y, Sun Y, et al: Hepatitis D virus infection of mice expressing human sodium taurocholate co-transporting polypeptide. PLoS Pathog 11: e1004840, 2015.

6. Ni Y, Lempp FA, Mehrle S, Nkongolo S, Kaufman C, Fälth M, Stindt J, Königer C, Nassal M, Kubitz R, et al: Hepatitis B and D viruses exploit sodium taurocholate co-transporting polypeptide for species-specific entry into hepatocytes. Gastroenterology 146: 1070-1083, 2014

7. Yuan W, Wu JY, Zhao YZ, Li J, Li JB, Li ZH and Li CS: Comparison of early sequential hypothermia and delayed hypothermia on neurological function after resuscitation in a swine model. Am J Emerg Med 35: 1645-1652, 2017.

8. Zhou M, Zhao F, Li J, Cheng Z, Tian X, Zhi X, Huang Y and $\mathrm{Hu}$ K: Long-term maintenance of human fetal hepatocytes and prolonged susceptibility to HBV infection by co-culture with non-parenchymal cells. J Virol Methods 195: 185-193, 2014.

9. Zhou M, Huang Y, Cheng Z, Zhao F, Li J, Zhi X, Tian X, Sun W and $\mathrm{Hu} \mathrm{K}$ : Revival, characterization, and hepatitis B virus infection of cryopreserved human fetal hepatocytes. J Virol Methods 207: 29-37, 2014.

10. Zhou M, Zhao K, Yao Y, Yuan Y, Pei R, Wang Y, Chen J, $\mathrm{Hu} X$, Zhou Y, Chen X and Wu C: Productive HBV infection of well-differentiated, hNTCP-expressing human hepatoma-derived (Huh7) cells. Virol Sin 32: 465-475, 2017. 
11. Ladner SK, Otto MJ, Barker CS, Zaifert K, Wang GH, Guo JT, Seeger $C$ and King RW: Inducible expression of human hepatitis B virus (HBV) in stably transfected hepatoblastoma cells: A novel system for screening potential inhibitors of HBV replication. Antimicrob Agents Chemother 41: 1715-1720, 1997.

12. Köck J, Nassal M, MacNelly S, Baumert TF, Blum HE and von Weizsäcker F: Efficient infection of primary tupaia hepatocytes with purified human and woolly monkey hepatitis B virus. J Virol 75: 5084-5089, 2001.

13. Köck J, Rösler C, Zhang JJ, Blum HE, Nassal M and Thoma C: Generation of covalently closed circular DNA of hepatitis B viruses via intracellular recycling is regulated in a virus specific manner. PLoS Pathog 6: e1001082, 2010.

14. Glebe D, Aliakbari M, Krass P, Knoop EV, Valerius KP and Gerlich WH: Pre-s1 antigen-dependent infection of Tupaia hepatocyte cultures with human hepatitis B virus. J Virol 77: 9511-9521, 2003.

15. Doitsh $\mathrm{G}$ and Shaul Y: A long HBV transcript encoding $\mathrm{pX}$ is inefficiently exported from the nucleus. Virology 309: 339-349, 2003.

16. Livak KJ and Schmittgen TD: Analysis of relative gene expression data using real-time quantitative PCR and the 2(-Delta Delta C(T)) Method. Methods 25: 402-408, 2001.

17. Nassal M: The arginine-rich domain of the hepatitis B virus core protein is required for pregenome encapsidation and productive viral positive-strand DNA synthesis but not for virus assembly. J Virol 66: 4107-4116, 1992.

18. Fushan AA, Turanov AA, Lee SG, Kim EB, Lobanov AV, Yim SH, Buffenstein R, Lee SR, Chang KT, Rhee H, et al: Gene expression defines natural changes in mammalian lifespan. Aging Cell 14: 352-365, 2015.

19. Drexler JF, Geipel A,König A, Corman VM, van RielD,Leijten LM, Bremer CM, Rasche A, Cottontail VM, Maganga GD, et al: Bats carry pathogenic hepadnaviruses antigenically related to hepatitis $B$ virus and capable of infecting human hepatocytes. Proc Nat Acad Sci USA 110: 16151-16156, 2013.
20. Vieira YR, dos Santos DR, Portilho MM, Velloso CE, Arissawa M, Villar LM, Pinto MA and de Paula VS: Hepadnavirus detected in bile and liver samples from domestic pigs of commercial abattoirs. BMC Microbiol 14: 315, 2014.

21. Li W, She R, Liu L, You H and Yin J: Prevalence of a virus similar to human hepatitis B virus in swine. Virol J 7: 60, 2010.

22. Tang H, Zhou S, Zhao L, Wang J, Lei B and Cao Z: Primary serologic investigation of HBV-like virus existence in domestic animals and fowls. Hua Xi Yi Ke Da Xue Xue Bao 21: 181-184, 1990 (In Chinese).

23. Ye X, Zhou M, He Y, Wan Y, Bai W, Tao S, Ren Y, Zhang X, Xu J, Liu J, et al: Efficient inhibition of hepatitis B virus infection by a preS1-binding peptide. Sci Rep 6: 29391, 2016.

24. Glebe D, Urban S, Knoop EV, Cag N, Krass P, Grün S, Bulavaite A, Sasnauskas K and Gerlich WH: Mapping of the hepatitis B virus attachment site by use of infection-inhibiting preS1 lipopeptides and tupaia hepatocytes. Gastroenterology 129: 234-245, 2005.

25. Doong SL, Tsai CH, Schinazi RF, Liotta DC and Cheng YC: Inhibition of the replication of hepatitis B virus in vitro by 2',3'-dideoxy-3'-thiacytidine and related analogues. Proc Natl Acad Sci USA 88: 8495-8499, 1991.

26. Joziasse DH and Oriol R: Xenotransplantation: The importance of the Galalpha1,3Gal epitope in hyperacute vascular rejection. Biochim Biophys Acta 1455: 403-418, 1999.

27. Lempp FA, Wiedtke E, Qu B, Roques P, Chemin I, Vondran FWR, Le Grand R, Grimm D and Urban S: Sodium taurocholate cotransporting polypeptide is the limiting host factor of Hepatitis B Virus infection in macaque and pig hepatocytes. Hepatology 66: 703-716, 2017.

This work is licensed under a Creative Commons Attribution-NonCommercial-NoDerivatives 4.0 International (CC BY-NC-ND 4.0) License. 